the work on rice at Hiratuka, on the morning glory at Misima, and the series of exhibits on wheat, radish and goldfish at Kyoto, will have failed to carry away a lasting impression of the peculiar strengths of Japanese genetics. Nor shall we easily forget the seedless water-melons offered us at the Institute organized by Dr. Kihara, their originator, at Yokohama.

Of the more social entertainments, which, as has been already mentioned, played a very important part in the general success of the meeting, some may have been most impressed by the visit to a Kabuki theatre, others perhaps by the trip to the famous architectural show-piece at Nikko. Personally, I think I shall treasure even more the dinner offered us on the first evening at a restaurant named the Hosigaoka-saryo. The banal word 'restaurant' conveys little of the reality of this place. The establishment is, more nearly, a park extending up a steep hill below which the lights of Tokyo stretch glittering towards the horizon. Perched on ledges of the hill are temporary marquees, and permanent pavilions with interiors of the fresh purity of line and colour characteristic of classical Japanese building. In one of these there was Japanese dancing, in another Chinese dishes were to be had, but the centre of attraction was a large terrace at which we were offered an initiation, as thorough as one wished to make it, into the delights of the Japanese cuisine. Attempts to master the trick of managing hachi (chopsticks), to identify the delicious but unexpected items of sea-food in the deep-fried fritters known as tempura, or the algae or other materials used for wrapping and flavouring the rice balls called sushi, to discuss whether the ricewine sake is nicer warm or cold, and if it is really quite strong or not strong at all-such things provided the perfect recipe for breaking the ice on the first day of what proved one of the most pleasant and valuable occasions of international contact which has been held in the biological world for many years.

C. H. WAdDington

\section{USE OF ANTIBIOTICS AGAINST PLANT DISEASES}

$\mathrm{T}$ HF title gives the theme of a symposium held on September 28 by the Association of Applied Biologists. The symposium, of eight papers, was mainly devoted to the action of two antibiotics, streptomycin and griseofulvin. The latter, a fungistatic substance produced by Penicillium spp., has been used solely against plant diseases. Streptomycin produced by Streptomyces spp., on the other hand, was originally used in medical research and has only recently been extensively tested against bacterial diseases of plants.

In some of the experiments discussed, the effects of these antibiotics might have been caused by their action at sites remote from the point of applicationa property which contrasts with the action of contact fungicides at or near the point of application only.

In his paper, Mr. S. H. Crowdy (Jealott's Hill Research Station) gave proof that both these antibiotics are absorbed from culture solutions by roots of uninfected plants, and that the amount of griseofulvin taken up is simply related to the amount of water transpired and the concentration in the solution. Once within the plant, their fates vary from one host spectes to another. The greater pro- portion of griseofulvin taken up by tomatoes stays in the roots, whereas in broad beans the majority moves from the roots and is found in the lower leaves; little is found in the stems of either plant. In contrast, streptomycin accumulates freely in tomato shoots, but only traces are found in the base of broad bean shoots even after prolonged treatment. Similarly, Lelliott found that streptomycin moves only very slowly from the base of carnation cuttings, and even though the cuttings were in streptomycin solutions containing 100 p.p.m. for only $24 \mathrm{hr}$., its presence could still be detected after 73 days. The rate of decay of griseofulvin in broad beans is relatively constant-there is a straight-line relation between the logarithm of the percentage griseofulvin surviving and time; 50 per cent of the griseofulvin disappears within 5 days.

Griseofulvin affects the development of a wide range of fungal and allied diseases, for example, those caused by Plasmodiophora brassicae, Fusarium spp., Erysiphe spp. and Botrytis spp. Adding griseofulvin to mixtures of unsterilized soil and sand inoculated with resting spores of $P$. brassicae decreases the number of root-hair infections on cabbages, the percentage plants clubbed and the size, at least temporarily, of the clubbed roots. In common with the standard fungicides, griseofulvin is most effective against low concentrations of inoculum. Where griseofulvin is only sufficient to reduce club size temporarily, eventually, when its retarding effect has disappeared, bigger clubs are produced than on the untreated plants. Associated with the larger clubs is a greater weight of foliage. This result probably reflects the ability of large plants when inoculated--in this experiment equivalent to when the griseofulvin no longer inhibited-to develop bigger clubs with less disruption than small plants.

The effects of soil applications of griseofulvin given three weeks after inoculation suggest that it can act systemically against $P$. brassicae. By then infection had occurred and the early stages of club formation within the root cortex were probably taking place. A week later clubs were seen only on the untreated controls, and after a further four weeks the clubs on the untreated controls were still five times larger than on the treated plants (Drs. F. T. Last and I. Macfarlane, Rothamsted Experimental Station).

Further evidence of its systemic properties was given by Dr. A. Rhodes and Messrs. R. Crosse and R. MeWilliam (Glaxo Laboratories, Ltd.), who controlled chrysanthemum powdery mildew, a leaf disease caused by Oidium chrysanthemi, by watering the roots with a wettable powder formulation of griseofulvin. Although no attempt was made to give equal amounts of active principle, soil applications were more effective than foliage sprays. Sprays containing 0.025 per cent griseofulvin were as effective as 'karathane' (dinitro capryl phenyl crotonate) sprays containing 0.05 per cent active principle.

Two sprays containing $0 \cdot 025$ per cent griseofulvin, one prepared with a wettable powder and the other with an NN-dimethylformamide liquid concentrate, were equally effective against chrysanthemum powdery mildew. The efficacy of the griseofulvin, however, was less in the wettable powder formulation since the powder alone gave significantly greater control of mildew than NN-dimethylformamide. In other small plot tests, griseofulvin controlled the incidence of Botrytis cinerea on lettuce and B. tulipae on tulips. In the control of the latter disease, tulip 
fire, under commercial conditions, griseofulvin sprays were more effective than 'Ferbam' sprays (ferric dimethyl dithiocarbamate) when compared weight for weight of active principle.

The results of experiments done in artificial and natural conditions do not invariably agree. Mr. J. Drew Smith (Sports Turf Research Institute) has found in preliminary trials that spray applications $(0 \cdot 044-0.088$ per cent griseofulvin) to turf, before inoculation with Fusarium nivale and Sclerotinia homoeocarpa, give very effective control of their respective diseases, F'usarium patch and dollar spot. In field trials relying on natural infection, however, they appear less effective and do not give as good control as mercury and cadmium fungicides. The apparent drop in the efficiency of griseofulvin may be attributable to changes in its formulation.

Similarly, the field-results using streptomycin do not always confirm the promise shown by initial tests. Of the two materials, streptomycin and 'Agrimycin' (1:10 mixture of oxytetracycline and streptomycin), the former was used against Pseudomonas caryophylli, the cause of bacterial wilt of carnations, and the latter against Erwinia sp., the cause of slow wilt of carnations. The highest minimal bactericidal dose recorded for a range of isolates was 5 p.p.m. of streptomycin against Ps. caryophylli and 3 p.p.m. of 'Agrimycin' against Erwinia sp.

The effects of these antibiotics on their respective diseases vary with the substrate in which the crops are, when they are applied. By standing a mixture of healthy and infected cuttings in solutions of 50-100 p.p.m. for $24 \mathrm{hr}$., both materials eliminate transmission but do not cure the infected plants. However, solutions of 'Agrimycin' of similar concentrations do not significantly reduce the spread of slow wilt when watered to cuttings rooting in sand.

The discrepancy between the effective bactericidal doses of both streptomycin and 'Agrimycin' against their respective pathogens in culture and on the host may be attributed to many factors. As with some of the standard fungicides, for example, chloronitrobenzenes, the efficacy of the antibiotics may be affected by the substrate on which the test organism is growing (Mr. R. A. Lelliott, Plant Pathology Laboratory, Ministry of Agriculture, Fisheries and Food).

The effect of streptomycin on bacterial canker of cherry caused by Pseudomonas mors-prunorum varies with the stage in the host's development at application. Spraying trees with streptomycin hydrochloride (220 p.p.m.) during the spring blossom period has a large immediate effect in reducing the severity of the necrotic leaf-spotting phase of the disease, but has little persistent effect on the supply of inoculum available for infection of branches in the autumn, as measured by the numbers of bacteria present on the leaf surfaces. Autumn sprays of streptomycin reduce the amount of leaf-surface inoculum, but less effectively than 'low'-strength Bordeaux mixtures. However, despite its apparent inferiority as a surface bactericide, streptomycin is almost as effective in reducing branch infections as Bordeaux mixture. Unequivocal evidence of the systemic activity of streptomycin was not obtained, although the effects of the spring sprays for the control of the leaf-spot phase may be partly attributed to this mode of action (I)r. J. E. Crosse, East Malling Research Station).

At this symposium, there was ample evidence to show that griseofulvin and streptomycin can reduce the severity of a range of plant diseases, and in some trials, notably against chrysanthemum powdery mildew, tulip fire and the silvering disease of beet, the antibiotics were at least as effective as, if not more effoctive than, the materials at present in general use. The sced-borne organism causing silvering disease of beet, Corynebacterium betue, was as effectively controlled by soaking seeds in streptomycin solutions at 200 p.p.m. for $24 \mathrm{hr}$. as by dusting them with mercury fungicides (Dr. W. G. Keyworth, National Vegetable Research Station).

'To gain the maximum advantage from these materials, emphasis should be turned to studying their accumulation within the host in relation to possible disease control. Thus with tomatoes, which accumulate griseofulvin in the roots, the probability of control is greater with root- than with leaf-infecting parasites.

Their use, as ,with most chemicals, does, however, present a number of hazards. Concentrations of griseofulvin unnecessarily high for the control of clubroot damaged cabbages, and the root growth of carnation cuttings, stood for $24 \mathrm{hr}$. in streptomycin solutions, progressively decreased as the concentration increased above 50 p.p.m. Rooting hormone can, however, be used as an antidote to this latter effect.

Apart from their harmful effects to plants when used indiscriminately, they may in addition detrimentally affect the health of man, a fact stressed by Dr. R. A. Shooter (St. Bartholomew's Hospital, London). In medical practice, some people, principally those dealing daily with its administration, have developed a sensitivity to streptomycin, and so cannot now avail themselves of its beneficial properties against disease unless de-sensitized. This risk has since been much reduced by making simple alterations in the handling technique. Apart from the preparation of sprays, the risk of developing sensitivity to antibiotics, if used as plant protectants, may arise from exposure to spray drift. It is, how: ever, probably much less than in medicine, since the concentrations used are much lower. The effects described of streptomycin against plant diseases were caused by 0.02 per cent solutions; in medicine, preparations containing 10 per cent are used. Al. though no examples of sensitization from handling antibiotics included in animal feeding stuffs were quoted during the symposium, the effect of consuming residues persisting on and in sprayed food is still a matter of conjecture and needs investigating.

These two hazards, toxicity to plants and sensitization of man, can be minimized by $(a)$ thorough research before making recommendations and $(b)$ by careful handling techniques. Already streptornycin is being used against plant diseases in New Zealand and the United States. A possible third hazard, the development of parasites resistant to antibiotics, is, however, less readily combated. Although the minimal dose of a material necessary to kill or inhibit the growth of parasites in vitro may be known, it is impossible to maintain such concentrations continuously in vivo. Thus conditions occur in which the concentrations of antibiotics are sub-lethal or sub-inhibitory, and in which adjustments in the population of parasites may take place. Although this type of adaptation has not been a serious problem in the control of plant pathogens with standard fungicides in the field, in medicine, and especially with the antibiotics, it causes great difficulties. As yet, antibiotics have been used against plant disease in the field for too short a time to assess the magnitude of this hazard.

F. T. LAST 